\title{
Glycosaminoglycan-functionalized poly-lactide-co- glycolide nanoparticles: synthesis, characterization, cytocompatibility, and cellular uptake
}

This article was published in the following Dove Press journal:

International Journal of Nanomedicine

19 January 2015

Number of times this article has been viewed

\section{Surya P Lamichhane' \\ Neha Arya ${ }^{1,2}$ \\ Nirdesh Ojha ${ }^{3}$ \\ Esther Kohler' \\ $\checkmark$ Prasad Shastri ${ }^{1,2,4}$}

'Institute for Macromolecular Chemistry, University of Freiburg, Freiburg, ${ }^{2} \mathrm{Helmholtz}$ Virtual Institute on "Multifunctional Biomaterials for Medicine", "Laboratory for Process Technology, Department of Microsystems Engineering, University of Freiburg, Freiburg, ${ }^{4}$ Centre for Biological Signaling Studies (BIOSS), University of Freiburg, Freiburg, Germany
Correspondence: $\vee$ Prasad Shastri Institute for Macromolecular Chemistry, University of Freiburg, Stefan-Meier Str 3I, Hermann Staudinger Haus, Freiburg D-79104, Germany

Tel +49 76I 2036268

Fax +49 76I 2036306

Email prasad.shastri@gmail.com
Abstract: The efficient delivery of chemotherapeutics to the tumor via nanoparticle (NP)-based delivery systems remains a significant challenge. This is compounded by the fact that the tumor is highly dynamic and complex environment composed of a plurality of cell types and extracellular matrix. Since glycosaminoglycan (GAG) production is altered in many diseases (or pathologies), NPs bearing GAG moieties on the surface may confer some unique advantages in interrogating the tumor microenvironment. In order to explore this premise, in the study reported here polylactide-co-glycolide (PLGA) NPs in the range of 100-150 nm bearing various proteoglycans were synthesized by a single-step nanoprecipitation and characterized. The surface functionalization of the NPs with GAG moieties was verified using zeta potential measurements and X-ray photoelectron spectroscopy. To establish these GAG-bearing NPs as carriers of therapeutics, cellular toxicity assays were undertaken in lung epithelial adenocarcinoma (A549) cells, human pulmonary microvascular endothelial cells (HPMEC), and renal proximal tubular epithelial cells. In general NPs were well tolerated over a wide concentration range $(100-600 \mu \mathrm{g} / \mathrm{mL})$ by all cell types and were taken up to appreciable extents without any adverse cell response in A549 cells and HPMEC. Further, GAG-functionalized PLGA NPs were taken up to different extents in A459 cells and HPMEC. In both cell systems, the uptake of heparin-modified NPs was diminished by $50 \%-65 \%$ in comparison to that of unmodified PLGA. Interestingly, the uptake of chondroitin sulfate NPs was the highest in both cell systems with $40 \%-60 \%$ higher uptake when compared with that of PLGA, and this represented an almost twofold difference over heparin-modified NPs. These findings suggest that GAG modification can be explored as means of changing the uptake behavior of PLGA NPs and these NP systems have potential in cancer therapy.

Keywords: tumor microenvironment, nanocarriers, proteoglycans, polysaccharides, passive targeting

\section{Introduction}

Second only to heart disease, cancer accounts for nearly $13 \%$ of all deaths in the USA. ${ }^{1}$ In spite of significant advances in the detection of solid tumors and treatment options, the efficient delivery of anticancer agents to the target site still remains a primary challenge. ${ }^{2}$ Over the past decade, nanoparticle (NP)-based delivery systems have gained importance in drug-delivery applications and demonstrated potential in the tissue-targeted delivery of cancer therapeutics. ${ }^{3}$ Cancer therapeutics can be targeted either by passive or active targeting. "Passive targeting" utilizes the changes to permeability of vasculature associated with tumor environment, which promotes the diffusion and accumulation of macromolecules and drugs by "extravasation", the enhanced permeation and retention effect to localize the therapy to the tumor environment. ${ }^{4}$ In this regard, NPs offer several advantages due to their size and ability to present 
cell- or tissue-specific moieties on their surface. However, following intravenous administration, the majority of the NPs end up in the liver and this has provided the impetus for developing strategies to enhance localization of therapy to tumors through active targeting. 5,6 "Active targeting" is primarily based on ligand-receptor interactions between the receptors overexpressed by cancer cells and ligand-modified NPs that then promote affinity for cancer cells within solid tumor. ${ }^{7,8}$ This approach has many merits but presents a few challenges with regard to ensuring that the targeting ligand is expressed constitutively in all grades and stages of the tumor. Since NP biodistribution is influenced by protein adsorption, and extracellular matrix (ECM) polysaccharides have specific affinity for proteins, the functionalization of the NP surface with ECM polysaccharides or proteoglycans may present new avenues to tissue targeting, based on proteinECM interactions.

The objective of the work reported here was therefore to synthesize and characterize poly-lactide-co-glycolide (PLGA) NPs bearing glycosaminoglycans (GAGs), namely heparin $(\mathrm{H})$, hyaluronic acid (HA), chondroitin sulfate (CS), and dermatan sulfate (DS). As preliminary screening for establishing the safety of these GAG-modified NPs for injectable therapy, the toxicity of these in lung epithelial adenocarcinoma (A549), human pulmonary microvascular endothelial cells (HPMEC), and human renal proximal tubular epithelial cells (RPTEC) was studied by 3-(4,5-dimethylthiazol-2-yl)-2,5-diphenyltetrazolium bromide (MTT) assay, lactate dehydrogenase (LDH) release, and induction of reactive oxygen species (ROS). In addition, to validate this system for the treatment of lung cancer, the uptake behavior of these NPs in A549 cells and HPMEC was evaluated.

\section{Materials and methods Chemicals}

Chondroitin-4-sulfate sodium salt from bovine trachea, DS from procaine intestinal mucosa, HA sodium salt from Streptococcus equi, H sodium salt derived from bovine intestinal mucosa, and PLGA, 50:50 (number average molecular weight $\left[\mathrm{M}_{\mathrm{w}}\right]$ 24,000-38,000 $\mathrm{g} / \mathrm{mol}$ ) were purchased from Sigma-Aldrich Co (St Louis, MO, USA). Acetone was procured from VWR International (Radnor, PA, USA) and tetrahydrofuran (THF) was procured from Carl Roth $\mathrm{GmbH}$ (Karlsruhe, Germany).

\section{Nanoparticle preparation and characterization}

GAG-functionalized NPs were synthesized by nanoprecipitation using a previously described single-step synthesis. ${ }^{9}$ Briefly, a $10 \mathrm{mg} / \mathrm{mL}$ solution of PLGA was prepared in a binary solvent system containing THF and acetone $(9: 1 \% \mathrm{v} / \mathrm{v})$, and the aqueous phase containing the watersoluble polyelectrolyte GAG at the desired concentration of $0.45 \mathrm{mg} / \mathrm{mL}$ was prepared in in deionized water. NP formation was induced by the rapid addition of $1 \mathrm{~mL}$ of aqueous phase to an equal volume of organic phase. The resulting suspension was dialyzed against deionized water, which was replaced every 2 hours, for a total of 8-12 hours.

For preparation of fluorescently labeled NPs, $0.2 \mathrm{mg} / \mathrm{mL}$ of Rhodamine-B base (Sigma-Aldrich Co) was additionally introduced to the organic phase. NP size and polydispersity index (PDI) were measured by dynamic light scattering (DLS) using a Delsa ${ }^{\text {TM }}$ Nano C (Beckman Coulter Inc, Brea, CA, USA) with a scattering angle of $165^{\circ}$ at room temperature. At least three measurements $(n=3)$ were taken and values reported as the average \pm standard deviation (SD).

Zeta potential was measured in triplicate using the Delsa Nano $\mathrm{C}$ with a scattering angle of $15^{\circ}$ at room temperature in deionized water, phosphate-buffered saline, and cell-culture medium, and the values reported as the average \pm SD.

The morphology of the GAG-functionalized PLGA NPs was determined by transmission electron microscopy (TEM). Briefly, the NP suspension was stained with uranyl formate placed on TEM grid and then air dried before analyzing using a Carl Zeiss LEO 912 Omega microscope (Oberkochen, Germany).

\section{Confirmation of surface functionalization by $\mathrm{pH}$ titration}

The zeta potential of the GAG-modified NPs was determined as a function of $\mathrm{pH}$ using the Delsa Nano C equipped with a pH auto-titrator. Briefly, the zeta potential of the diluted NP suspension was measured at room temperature at $\mathrm{pHs} 2,3$, 5 , and 7. The data were then used to calculate the isoelectric point $\left(\mathrm{p} I_{\mathrm{e}}\right)$ of the NP surface and this was compared with the $\mathrm{p} I_{\mathrm{e}}$ of the respective functional moiety.

\section{X-ray photoelectron spectroscopy}

$\mathrm{X}$-ray photoelectron spectroscopy (XPS) was used to confirm the surface functionalization of the PLGA NPs with GAGs. The primary objective of the investigation was to identify the presence of H, HA, DS, and CS on the surface of the functionalized PLGA NPs. For this purpose, a widescan spectrum of each of the samples was obtained and corrected for background noise. The X-ray photoelectron spectra of the samples for carbon $1 \mathrm{~s}(\mathrm{C} 1 \mathrm{~s})$ and oxygen $1 \mathrm{~s}$ (O1s) were obtained at a higher resolution (narrow scan) for detailed analysis. Since the surfaces of the samples were nonconductive, the experimental binding energies were 
corrected for charge-induced shifts in binding energy using the charge correction factor. First the peaks were fitted for the $\mathrm{O} 1 \mathrm{~s}$ and the numerical value of the binding energy of the component peak was determined. This numerical value was then subtracted from the reference value of the $\mathrm{O} 1 \mathrm{~s}$ ' binding energy as found in the literature to obtain the charge correction factor. ${ }^{10}$ The X-ray photoelectron spectra for $\mathrm{C} 1 \mathrm{~s}$ were then de-convoluted using OriginPro software (v 9.8G; OriginLab Corporation, Northampton, MA, USA). The cumulative curve for each of the samples had an adjusted $R^{2}$ value better than 0.995 .

\section{Cell-culture experiments}

A549 cells were obtained from the Center for Biological Signaling Studies (BIOSS) Toolbox, University of Freiburg (Germany) and cultured in Dulbecco's Modified Eagle's Medium supplemented with 10\% fetal bovine serum (Thermo Fisher Scientific, Waltham, MA, USA), and 1\% penicillin/streptomycin (P/S) (100 U/mL; PAN-Biotech GmbH, Aidenbach, Germany). HPMEC were procured from ScienCell Research Laboratories (Carlsbad, CA, USA) and grown in endothelial-cell growth medium (ScienCell) supplemented with $5 \%$ fetal bovine serum and $1 \% \mathrm{P} / \mathrm{S}$. RPTEC were purchased from Lonza Group (Basel, Switzerland) and were grown in renal-cell growth media (REBM basal medium, Lonza). All cells were maintained in a humidified incubator at $37^{\circ} \mathrm{C}$ with $5 \% \mathrm{CO}_{2}$.

\section{Cellular compatibility}

The effect of the GAG-modified PLGA NPs on the proliferation of A549 cells, HPMEC, and RPTEC was determined using MTT assay. Since the formation of the insoluble formazan product requires mitochondrial esterase, this assay is frequently used to ascertain the metabolic status of cells. Briefly, cells were plated in 96 -well plate $\left(6 \times 10^{3}\right.$ cells $/$ well $)$ and, 24 hours after plating, were treated with increasing concentrations of the functionalized NPs, with the MTT assay performed at the end of the 24 hours.

Briefly, the culture medium was replaced with $100 \mu \mathrm{L}$ MTT solutions $(0.25 \mathrm{mg} / \mathrm{mL})$ and the plates were incubated for 3 hours at $37^{\circ} \mathrm{C}$ and in $5 \% \mathrm{CO}_{2}$. Then, the MTT reagent from the wells was aspirated and $100 \mu \mathrm{L}$ dimethyl sulfoxide (DMSO) was added to each well to dissolve the purple formazan crystals. The cell viability was assessed by absorbance at $550 \mathrm{~nm}$ using a microplate reader (BioTek Instruments, Inc, Winooski, VT, USA). The cell viability is represented as the percentage with respect to untreated samples (control) and represents an average of at least three independent experiments $(n \geq 3)$.

\section{Lactate dehydrogenase assay}

The effect of the NPs on the integrity of the cell membrane was assayed using a LDH Cytotoxicity Assay Kit II (Abcam plc, Cambridge, England). The assay was performed as per the manufacturer's instructions. LDH is released by cells in response to damage or loss of integrity of cell membrane and is an indicator cellular toxicity. Briefly, $4 \times 10^{4}$ cells/well were plated in a 96-well plate and treated with the functionalized PLGA NPs for 24 hours. Untreated cells were taken as the negative control and cells treated with lysis buffer were taken as the high control (total LDH in the cell). As a positive control, $1 \mu \mathrm{L}$ of $\mathrm{LDH}$ was used to validate the assay. Following the incubation with NPs, the well plates were centrifuged at $600 \mathrm{~g}$ for 10 minutes and $10 \mu \mathrm{L}$ of the medium was transferred to a fresh 96-well plate. The medium was then incubated with $100 \mu \mathrm{L}$ of $\mathrm{LDH}$ reaction mixture for 15 minutes at room temperature and the absorbance was measured at $450 \mathrm{~nm}$ using the microplate reader (BioTek Instruments, Inc) with the reference wavelength at $650 \mathrm{~nm}$. LDH was quantified using the following formula:

$$
\mathrm{LDH} \%=\frac{\text { Test }- \text { low control }}{\text { High control }- \text { low control }} \times 100
$$

in which "low control" was the cells without any treatment and "high control" was the cells treated with lysis buffer (total LDH).

\section{Induction of intracellular reactive oxygen species by glycosaminoglycan-modified nanoparticles}

Intracellular ROS were determined using $2^{\prime}, 7^{\prime}$-dichlorofluorescein diacetate (DCFH-DA) (Abcam plc) using the protocol described by the supplier. DCFH-DA interacts with the intracellular ROS and gets converted to a fluorescent compound, dichlorofluorescein (DCF). The intensity of DCF is therefore directly proportional to intracellular ROS. Briefly, cells were plated in a 96 -well plate $\left(3 \times 10^{4}\right.$ cells/well) and exposed to GAG-functionalized and un-functionalized PLGA NPs for 24 hours. Following this, the cells were washed twice with phosphate-buffered saline (PBS) and then incubated with a working solution of DCFH-DA $(200 \mu \mathrm{L} /$ well $)$ at $37^{\circ} \mathrm{C}$ for 30 minutes. Fluorescence was then recorded at $485 \mathrm{~nm}$ excitation and $520 \mathrm{~nm}$ emission using the microplate reader (BioTek Instruments, Inc). Cells not exposed to NPs served as controls. Hydrogen peroxide $(50 \mu \mathrm{M})$ was used to validate 
the assay for each run. The ROS were quantified using the following formula:

$$
\mathrm{DCF} \%=\frac{\text { test }}{\text { control }} \times 100
$$

in which "control" was the cells without any treatment.

\section{Cellular uptake of NPs}

To investigate the cellular uptake of the functionalized PLGA NPs, cells were seeded in an eight-well chamber slide $\left(2 \times 10^{4}\right.$ cells/well). After 24 hours the cells were exposed to $200 \mu \mathrm{g} / \mathrm{mL}$ of surface-functionalized PLGA NPs and then incubated for 4 hours. The cells were then fixed with $4 \%$ paraformaldehyde and mounted with mounting media containing 4',6-diamidino-2-phenylindole (DAPI; Vectashield $^{\circledR}$, Vector Laboratories Inc, Burlingame, CA, USA). The fluorescence images were then taken using a Carl Zeiss microscope.

Further, for flow cytometry (fluorescence-activated cell sorting [FACS], $10 \times 10^{4}$ cells were seeded in a 12 -well plate. Following overnight attachment, the cells were treated with $200 \mu \mathrm{g} / \mathrm{mL}$ of GAG-functionalized PLGA NPs for 4 hours. Before performing FACS, the samples were washed with PBS in order to remove non-internalized NPs from the cell surface. The uptake efficiency was determined with a FACS analyzer (Gallios ${ }^{\mathrm{TM}}$ Flow Cytometer, Beckman Coulter Inc) considering 10,000 counts per sample $(n=3)$. The samples were analyzed using the Flowing Software (v 2.5.0; University of Turku, Turku, Finland).

\section{Statistical analysis}

The statistical analysis was performed using Student's $t$-test and $P$-values $<0.05$ were considered statistically significant. Error bars represent SDs.

\section{Results and discussion}

\section{Synthesis and characterization of GAG-} functionalized PLGA NPs

Four GAGs commonly found in the ECM of tumor tissue namely H, HA, CS, and DS - were selected for the surface modification of PLGA NPs due to their varied physicochemical characteristics and affinity for growth factors. ${ }^{11}$ The single-step nanoprecipitation method developed in our laboratory allows for the concomitant functionalization of NP during its formation and thus enables the rendering of a variety of biologically relevant information without resorting to multi-step synthesis for the generation of modified
NPs. Moreover, this method enables the rapid production of NPs with the desired size and functional properties without the need for complex polymer backbone synthesis steps. ${ }^{9}$

The choice of acetone to THF ratio of $9: 1(\mathrm{v} / \mathrm{v})$ as the binary solvent system to dissolve the polymer was based on an optimization study to get the target NP size of 100-150 nm. This size range is desirable as it is well-suited for cellular uptake, ${ }^{12}$ increased circulation time within the solid tumors, favors NP extravasation from vasculature, and reduces the likelihood of lung embolism. ${ }^{13,14}$ This latter attribute can augment the primary targeting modality to solid tumors, which is based on the phenomenon of enhanced permeation and retention effect. ${ }^{4}$

GAG-functionalized NPs were characterized in terms of their size and surface charge using DLS. NP size, determined using DLS, is plotted as a function of GAG concentration in Figure 1A. DLS measurements revealed that the particle sizes of H-, HA-, CS-, and DS-modified PLGA NPs in aqueous solutions were in the range of 100$160 \mathrm{~nm}$ with a narrow PDI of $<0.2$ in all cases except HA. The size of the GAG-functionalized NPs in aqueous solution was similar to the size of the non-functionalized PLGA NPs thereby demonstrating that the modification with GAGs yielded NPs with similar hydrodynamic radius. Furthermore, the increase in GAG concentration in the aqueous phase had no appreciable effect on NP size when the concentration was above $0.45 \mathrm{mg} / \mathrm{mL}$ except in the case of HA. It has been reported previously that aqueous-phase viscosity plays a crucial role in controlling NP size in the modified nanoprecipitation method and that there exits a linear correlation between aqueous-phase viscosity and NP size. ${ }^{9}$ Therefore, the unusual increase in size in the case of HA may be attributed to the greater viscosity of HA solutions at higher concentrations. Based on these observations, NPs produced in presence of a GAG concentration of $0.45 \mathrm{mg} / \mathrm{mL}$ were chosen for all the future studies as this particular concentration consistently resulted in NPs in the desired size range of 100-150 nm (Table $\mathrm{S} 1$ ).

The morphology and size distribution of NPs were also investigated using TEM (Figure 1B and Table S2). TEM analysis confirmed that the GAG-modified NPs had a spherical morphology and the particles were homogeneously distributed. However, the size distribution as obtained from TEM (Table S2) was lower and in the range of 80-125 nm. This is not surprising as DLS always overestimates size as it is based on hydrodynamic radius, which is very sensitive to surface chemistry. 


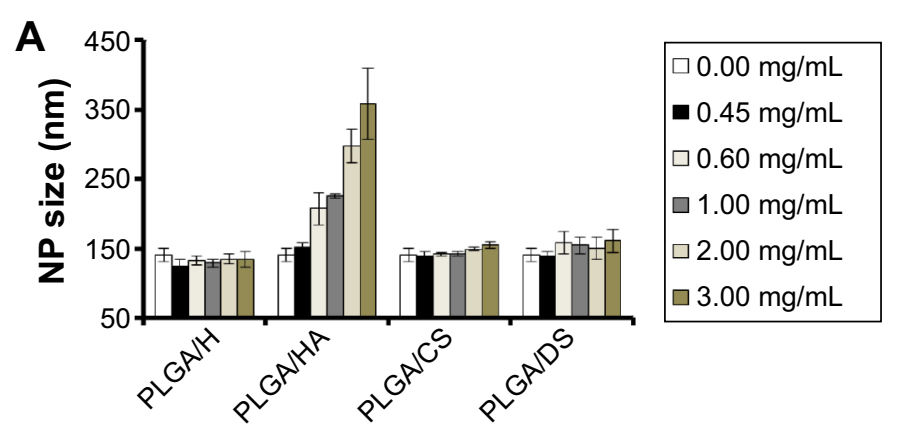

B
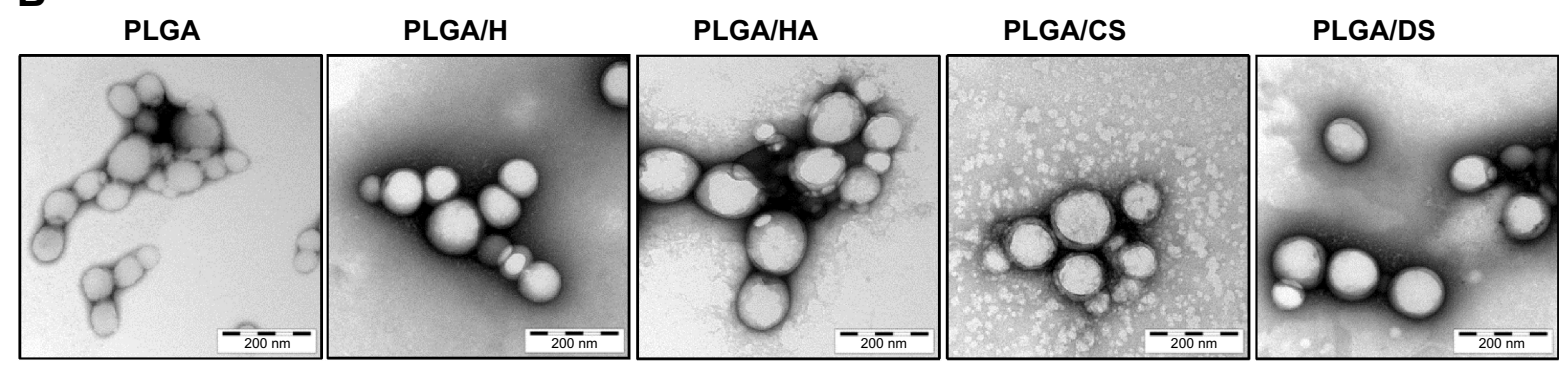

Figure I Effect of glycosaminoglycan (GAG) concentration on poly-lactide-co-glycolide (PLGA) nanoparticle (NP) size ( $\mathrm{n}=3$ ): (A) "0.00 mg/mL" corresponds to plain PLGA NPs; (B) transmission electron micrographs of GAG-functionalized PLGA NPs.

Abbreviations: PLGA/CS, poly-lactide-co-glycolide functionalized with chondroitin sulfate; PLGA/DS, poly-lactide-co-glycolide functionalized with dermatan sulfate; PLGA/H, poly-lactide-co-glycolide functionalized with heparin; PLGA/HA, poly-lactide-co-glycolide functionalized with hyaluronic acid.

\section{Confirmation of surface functionalization of poly-lactide-co-glycolide nanoparticles}

One of the primary objectives of this study was to establish the suitability of GAG-functionalized NPs for injectable therapeutics. Establishing the presence of GAG on the NP surface was therefore a key step in this direction. To confirm the presence of GAG modification on the PLGA NPs, two characterization strategies were employed: zeta potential measurements and XPS. In general, all GAG-functionalized NPs showed a large negative zeta potential ranging from -30 to $-40 \mathrm{mV}$ in deionized water, which represented a significant decrease when compared with that of unmodified PLGA NPs which was found to be $-21.66 \pm 1.50 \mathrm{mV}$ (Figure 2A). Since $\mathrm{pH}$ is related to the surface chemistry of the NP, the $\mathrm{p} I_{\mathrm{e}}$ of NP's surface can be used to confirm the nature of the ionizable moieties. A titration of zeta potential as a function of $\mathrm{pH}$ was undertaken (Figure 2B) and this revealed that the $\mathrm{p} I_{\mathrm{e}}$ values for each of the GAG-functionalized PLGA NPs closely matched the pKa of the dominant ionizable group associated with each respective GAG molecule (Table 1).

While the measurements strongly indicated the presence of GAG on the NP surface, direct confirmation of the surface chemistry was obtained using XPS. The processed C1S XPS spectra of PLGA (50:50) NPs (Figure 3A) showed three peaks: one at $289.1 \mathrm{eV}$, a second peak at $287.0 \mathrm{eV}$, and third peak at $285.1 \mathrm{eV}$ corresponding to the carbon atoms in $\mathrm{O}-\mathrm{C}=\mathrm{O}, \mathrm{C}-\mathrm{O}-\mathrm{C}$, and $\mathrm{C}-\mathrm{H}$ and $\mathrm{C}-\mathrm{C}$ bonds, respectively (Table S3). In addition to these three peaks, the XPS spectrum of all the functionalized NPs had varying intensity in the peak around $285 \mathrm{eV}$ and a shoulder at $285.5 \mathrm{eV}$. De-convolution of this peak confirmed the contribution from C-S and C-N bonds in the case of H, CS, and DS (Figure 3B, D, and E) and the contribution of $\mathrm{C}-\mathrm{N}$ in the case of HA (Figure 3C). This provides direct evidence for the presence of a GAG-rich surface in these NPs (Table 2).

\section{Cytotoxicity of glycosaminoglycan- functionalized poly-lactide-co-glycolide nanoparticles}

The in vitro cytotoxicity of the GAG-functionalized PLGA NPs was assessed on three different cell types: A549 cells, HPMEC and RPTEC. The rationale for the selection of these cell types was based on our interest in exploiting these functionalized PLGA NPs for targeting solid tumors like lung cancer and also in addressing the pulmonary and renal toxicity of GAG-modified PLGA NPs. HPMEC were primarily chosen as a model system to address the pulmonary toxicity of these NPs, as these cells form a selective barrier responsible for the exchange of gases and regulation of various fluids and solutes across the blood and cellular 

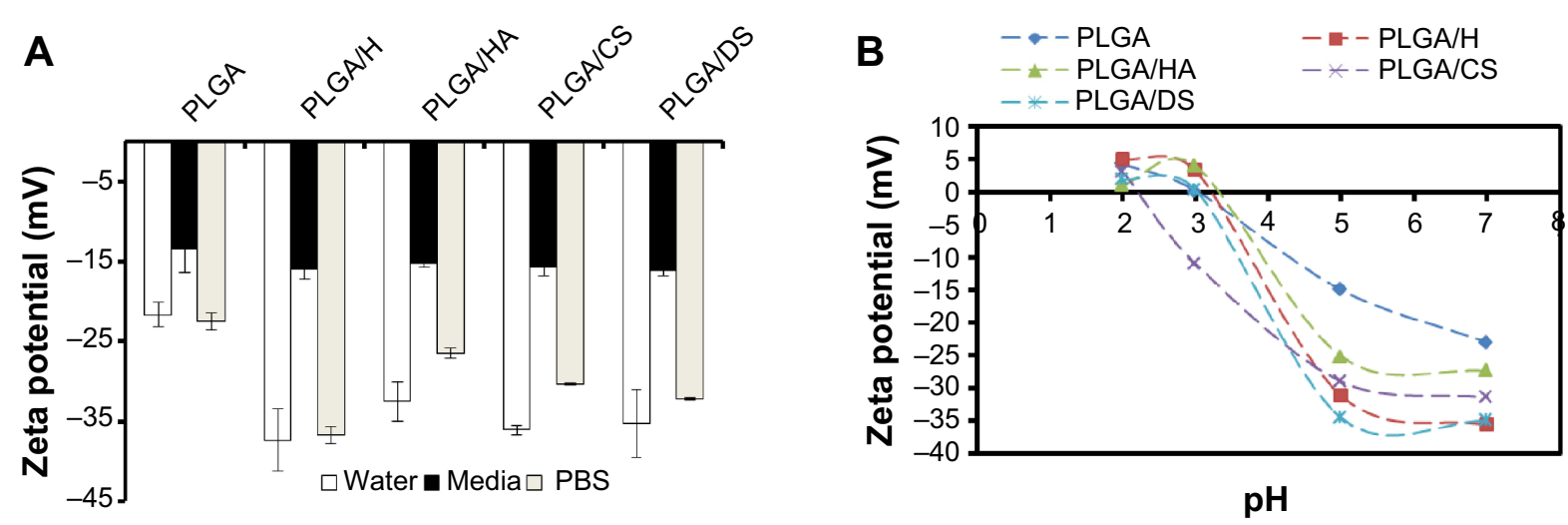

Figure 2 (A) Zeta potential measurement of glycosaminoglycan (GAG)-functionalized poly-lactide-co-glycolide (PLGA) nanoparticles (NPs) in deionized water, cell-culture medium, and phosphate-buffered saline $(\mathrm{pH} 7.4)(\mathrm{n}=3)$. (B) Isoelectric point measurement of the GAG-functionalized PLGA NPs as a function of $\mathrm{pH}$.

Abbreviations: PLGA/CS, poly-lactide-co-glycolide functionalized with chondroitin sulfate; PLGA/DS, poly-lactide-co-glycolide functionalized with dermatan sulfate; PLGA/H, poly-lactide-co-glycolide functionalized with heparin; PLGA/HA, poly-lactide-co-glycolide functionalized with hyaluronic acid.

compartments. ${ }^{15,16}$ Further, these cells have been reported to be prominent sites for the generation of ROS in the lungs during cellular injuries and inflammation. ${ }^{16,17}$ Additionally, RPTEC were chosen to address the renal toxicity of the functionalized NPs, as they have been commonly employed in understanding various renal diseases (eg, glomerulonephritis), injuries, inflammation, and toxicity..$^{18}$ Also, one of the concerns with long-term use of NP-based therapeutics is renal toxicity.

In the first set of experiments, the toxicity of surfacemodified PLGA NPs as a function of concentration was determined by MTT assay (Figure 4). It was observed that with an increase in the NP concentration (functionalized as well as non-functionalized), there was a moderate decrease in cell viability. A similar decrease in the viability of A549 cells at higher concentrations has been reported by Grabowski et al in a polyvinyl alcohol/chitosan-coated PLGA NP system. ${ }^{19}$ Also, Chung et al have reported 5\%-7\% toxicity in NIH/3T3 fibroblast and SCC7 tumor cells following incubation with $100 \mu \mathrm{g} / \mathrm{mL}$ of PLGA NPs for 24 hours. ${ }^{20}$ The increase in

Table I Surface characteristics of glycosaminoglycan-functionalized poly-lactide-co-glycolide (PLGA) nanoparticles (NPs)

\begin{tabular}{lllll}
\hline $\begin{array}{l}\text { NP } \\
\text { composition }\end{array}$ & $\begin{array}{l}\text { pKa of } \\
\text { surface } \\
\text { functionality }\end{array}$ & $\begin{array}{l}\text { pl }{ }_{\mathrm{e}} \text { of } \\
\text { surface }\end{array}$ & $\begin{array}{l}\text { Change in zeta } \\
\text { potential versus } \\
\text { unmodified } \\
\text { PLGA NPs }(\Delta \zeta)\end{array}$ & $\begin{array}{l}\text { Zeta } \\
\text { potential } \\
\text { at pH 7.0 }\end{array}$ \\
\hline PLGA & - & 2.9 & 0.0 & -23.0 \\
PLGA/H & $2.00-4.00$ & 3.2 & 14.5 & -35.7 \\
PLGA/HA & $3.00-4.00$ & 3.4 & 4.4 & -27.4 \\
PLGA/CS & $2.00-2.54$ & 2.2 & 8.5 & -31.5 \\
PLGA/DS & $2.00-6.00$ & 3.1 & 12.0 & -35.0
\end{tabular}

Abbreviations: $\mathrm{pl}$, isoelectric point; PLGA/CS, poly-lactide-co-glycolide functionalized with chondroitin sulfate; PLGA/DS, poly-lactide-co-glycolide functionalized with dermatan sulfate; PLGA/H, poly-lactide-co-glycolide functionalized with heparin; PLGA/HA, poly-lactide-co-glycolide functionalized with hyaluronic acid. toxicity at higher concentrations in all cases (including PLGA NPs) could be attributed to an increase in NP internalization by all cell types and not the surface moiety. The fact that the zeta potential of all four NPs decreased to values between -13.47 and $-16.12 \mathrm{mV}$ upon exposure to cell-culture medium (Figure 2A) is indicative of a partial neutralization of the surface charge following interaction with the serum proteins. ${ }^{21}$ Adsorbed serum proteins are expected to play a role in both cellular toxicity and uptake and this needs to be further investigated. Nevertheless, the results clearly indicate that the loss of cell viability was NP-concentration dependent as it was proportional to this variable rather than type of GAG, indicating that the surface functionalization was well tolerated by the cells.

While cell viability provides insights into the general cytocompatibility of an NP system, secondary processes triggered by exposure to and ingestion of nanomaterials may determine long-term outcomes. In this regard, the release of intracellular $\mathrm{LDH}$, which has been found to be associated with cellular necrosis, ${ }^{22}$ is a good indicator of cell-membrane integrity, and hence cellular toxicity induced by exposure to NPs. Assay for LDH in A549 cells, HPMEC, and RPTEC revealed that with increase in NP concentration, there was only a marginal increase in cellular toxicity (Figure 5). The increase in LDH was more prominent in HPMEC upon exposure to the GAG NPs, except in the case of DS-modified NPs, followed by A549 cells and RPTEC. Overall, the results indicated similar patterns of toxicity in all the experimental conditions and doses employed. The maximum cellular toxicity in all cells was observed at the highest NP concentration of $600 \mu \mathrm{g} / \mathrm{mL}$, which in HPMEC was observed following exposure to PLGA/ HA NPs and was $18.27 \% \pm 5.02 \%$, while the maximum cellular toxicity in A549 cells was similar (14.35\% $\pm 3.50 \%)$ following exposure to PLGA/H NPs, and in RPTEC the maximum 

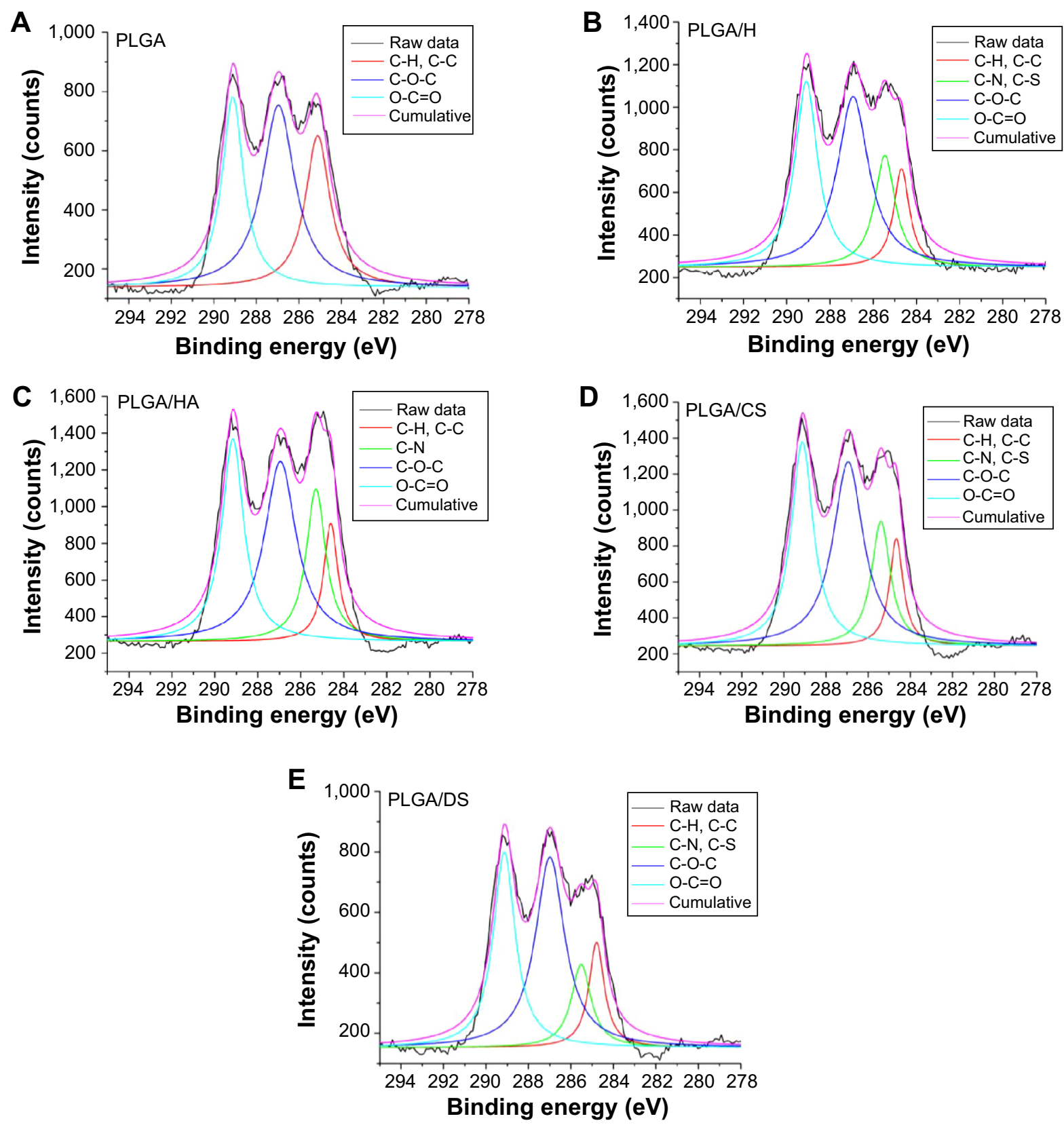

Figure 3 Cumulative carbon Is (Cls) X-ray photoelectron spectra of poly-lactide-co-glycolide (PLGA) nanoparticles bearing different glycosaminoglycans demonstrating binding energies of different glycosaminoglycans. Cls peaks at $289.1 \mathrm{eV}, 287 \mathrm{eV}$ and $285 . \mathrm{l} \mathrm{eV}$ correspond to $\mathrm{O}-\mathrm{C}=\mathrm{O}, \mathrm{C}-\mathrm{O}-\mathrm{C}$ and $\mathrm{C}-\mathrm{H}$ and $\mathrm{C}-\mathrm{C}$ bonds respectively in all samples. Cls peaks at $285 \mathrm{eV}$ and a shoulder at $285.5 \mathrm{eV}$ correspond to C-S and C-N in PLGA/H, PLGA/CS and PLGA/DS and correspond to C-N in PLGA/HA. Notes: X-ray photoelectron spectra of PLGA NPs: (A), PLGA; (B), PLGA/H; (C), PLGA/HA; (D), PLGA/CS; (E), PLGA/DS.

Abbreviations: PLGA/CS, poly-lactide-co-glycolide functionalized with chondroitin sulfate; PLGA/DS, poly-lactide-co-glycolide functionalized with dermatan sulfate; $\mathrm{PLGA} / \mathrm{H}$, poly-lactide-co-glycolide functionalized with heparin; PLGA/HA, poly-lactide-co-glycolide functionalized with hyaluronic acid.

of 13.22 \pm 3.69 was seen in presence of PLGA/H NPs. The generally higher cytotoxicity of these NPs in A549 cells and HPMEC might be attributed to their higher proliferative capacity, oxidative stress, and higher metabolic activity, while the increased cytotoxicity observed in HPMEC in the presence of PLGA/HA could be attributed to enhanced uptake of functionalized PLGA/HA NPs by HPMEC, as is discussed later.

It has been demonstrated in various mammalian cells that NP internalization induces an increased production of free oxygen radicals (also referred to as "ROS") leading to cellular stress and inflammation..$^{23}$ Therefore, as a third panel of cellular toxicity assessment in addition to MTT and LDH assays, ROS levels were determined (Figure 6). The induction of ROS in all the three cell types upon exposure to increasing concentrations of GAG-functionalized PLGA NPs was determined. It was observed that with an increase in GAG NP concentration, there was an increase in percentage DCF fluorescence indicative of an increase in ROS levels. This was consistent 
Table 2 Elemental composition of glycosaminoglycan-functionalized poly-lactide-co-glycolide (PLGA) nanoparticles (NPs) determined using $\mathrm{X}$-ray photon electron spectroscopy

\begin{tabular}{lllll}
\hline NP composition & Carbon & Nitrogen & Oxygen & Sulfur \\
\hline PLGA & 60.53 & 0.00 & 39.46 & 0.00 \\
PLGA/H & 60.12 & 0.09 & 39.74 & 0.14 \\
PLGA/HA & 61.37 & 0.19 & 38.37 & 0.00 \\
PLGA/CS & 60.19 & 0.24 & 39.42 & 0.06 \\
PLGA/DS & 58.62 & 0.08 & 40.04 & 0.15
\end{tabular}

Abbreviations: PLGA/CS, poly-lactide-co-glycolide functionalized with chondroitin sulfate; PLGA/DS, poly-lactide-co-glycolide functionalized with dermatan sulfate; PLGA/H, poly-lactide-co-glycolide functionalized with heparin; PLGA/HA, polylactide-co-glycolide functionalized with hyaluronic acid.

with what was already observed using the MTT and LDH assays. In A549 cells, the increase in ROS levels showed a dose dependency in all cases except in HA- and DS-functionalized NPs (Figure 6A). Interestingly, while PLGA/HA NPs triggered very low levels of ROS release, PLGA/DS NPs induced robust oxidative stress even at NP concentrations of $200 \mu \mathrm{g} / \mathrm{mL}$.
Similarly, in HPMEC, HA-modified NPs induced strong ROS levels even at a low concentration of $200 \mu \mathrm{g} / \mathrm{mL}$ (Figure 6B). The higher ROS levels in A549 cells on the other hand could be attributed to an increased state of oxidative stress in general in cancer cells due to the high degree of oncogenic transformation and increased metabolic activities. ${ }^{24-26}$ Our observation is corroborated by reports in the literature of an increase in production of ROS in both A549 cells and HPMEC after exposure to zinc oxide NPs. ${ }^{23,27}$

It is also well known that both A549 cells and HPMEC have an enhanced susceptibility toward ROS generation, ${ }^{24}$ and that lung microvascular endothelial cells are the primary sites for oxidative injury during the inflammation or injuries. ${ }^{16}$ Both of these factors might have played a role in the higher ROS production observed in these cells. Higher oxidative stress in A549 cells is advantageous, since it is known that ROS-generating agents like hydrogen peroxide ${ }^{28}$ and drug-delivery vehicles like
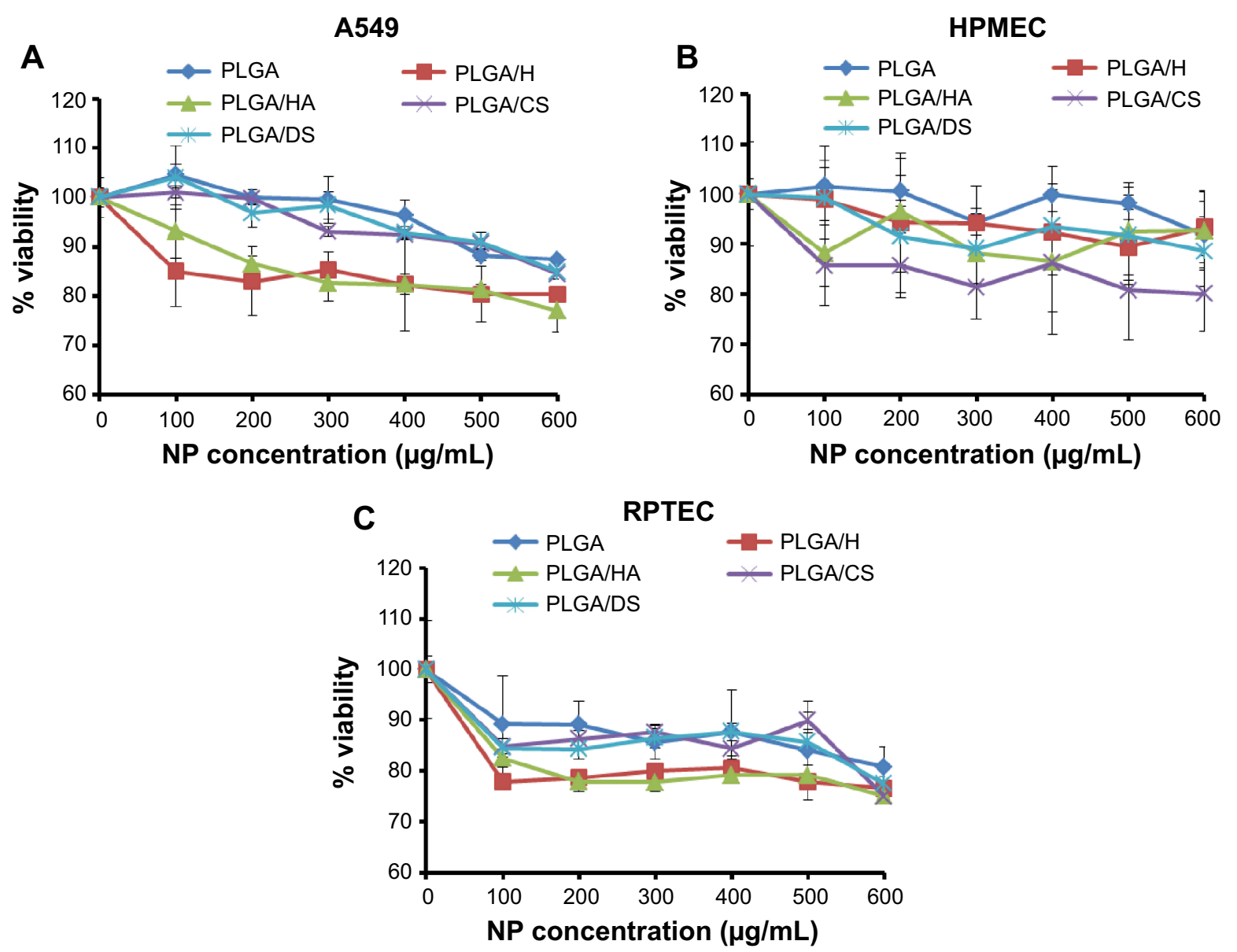

Figure 4 Viability (as a percentage of tissue-culture plastic control) of (A) lung epithelial adenocarcinoma (A549) cells, (B) human pulmonary microvascular endothelial cells (HPMEC), and (C) renal proximal tubular epithelial cells (RPTEC) following exposure to increasing concentrations of glycosaminoglycan-functionalized poly-lactide-coglycolide (PLGA) nanoparticles (NPs) for 24 hours ( $n=3$ ).

Abbreviations: PLGA/CS, poly-lactide-co-glycolide functionalized with chondroitin sulfate; PLGA/DS, poly-lactide-co-glycolide functionalized with dermatan sulfate; PLGA/H, poly-lactide-co-glycolide functionalized with heparin; PLGA/HA, poly-lactide-co-glycolide functionalized with hyaluronic acid. 


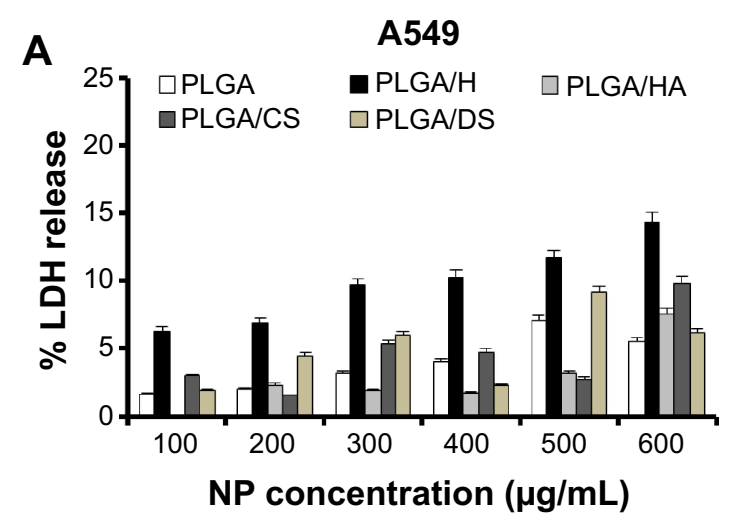

C

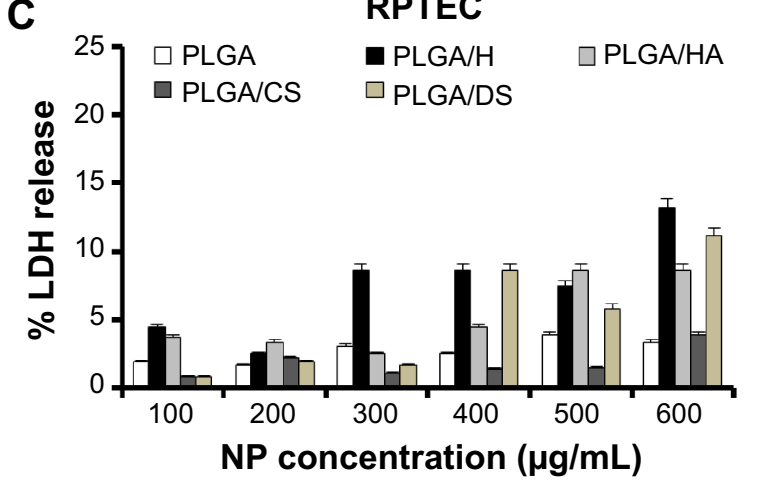

RPTEC

Figure 5 Effect of glycosaminoglycan-functionalized poly-lactide-co-glycolide (PLGA) nanoparticles (NPs) on cell-membrane integrity following exposure to increasing concentrations of GAG-functionalized PLGA NPs for 24 hours. The relative lactate dehydrogenase (LDH) release was co-related to percentage cytotoxicity. Cytotoxicity due to loss in membrane integrity in (A) lung epithelial adenocarcinoma (A549) cells, (B) human pulmonary microvascular endothelial cells (HPMEC), and (C) renal proximal tubular epithelial cells (RPTEC) $(n=3)$.

Abbreviations: PLGA/CS, poly-lactide-co-glycolide functionalized with chondroitin sulfate; PLGA/DS, poly-lactide-co-glycolide functionalized with dermatan sulfate; $\mathrm{PLGA} / \mathrm{H}$, poly-lactide-co-glycolide functionalized with heparin; PLGA/HA, poly-lactide-co-glycolide functionalized with hyaluronic acid.

carbon nanotubes and graphene oxide ${ }^{21}$ are potentiators for the enhanced toxicity of paclitaxel on cancer cells. In this regard, since GAG-functionalized PLGA NPs caused ROS production in A549 cells, they could be used as adjuvants for anticancer drugs like paclitaxel. In contrast, the ROS induction in RPTECs was significantly lower with a maximum of less than half of that with HPMECs and A549 cells, indicating a minimal level of oxidative stress in RPTEC as compared with A549 cells and HPMECs. This is an encouraging result, as renal toxicity is one of the concerns of long-term NP therapy.

Taken together, the results from the three toxicity screens indicate only a low-moderate level of cellular toxicity following exposure to GAG-functionalized PLGA NPs, over a wide concentration range.

\section{Cellular uptake of glycosaminoglycan- functionalized poly-lactide-co-glycolide nanoparticles}

Since the objective of the present study was to develop an NP platform to deliver therapeutics to lung tumors, preliminary

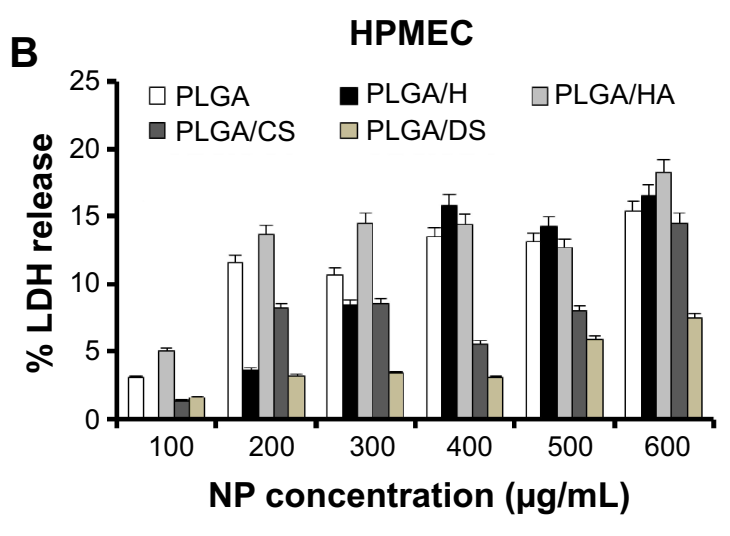



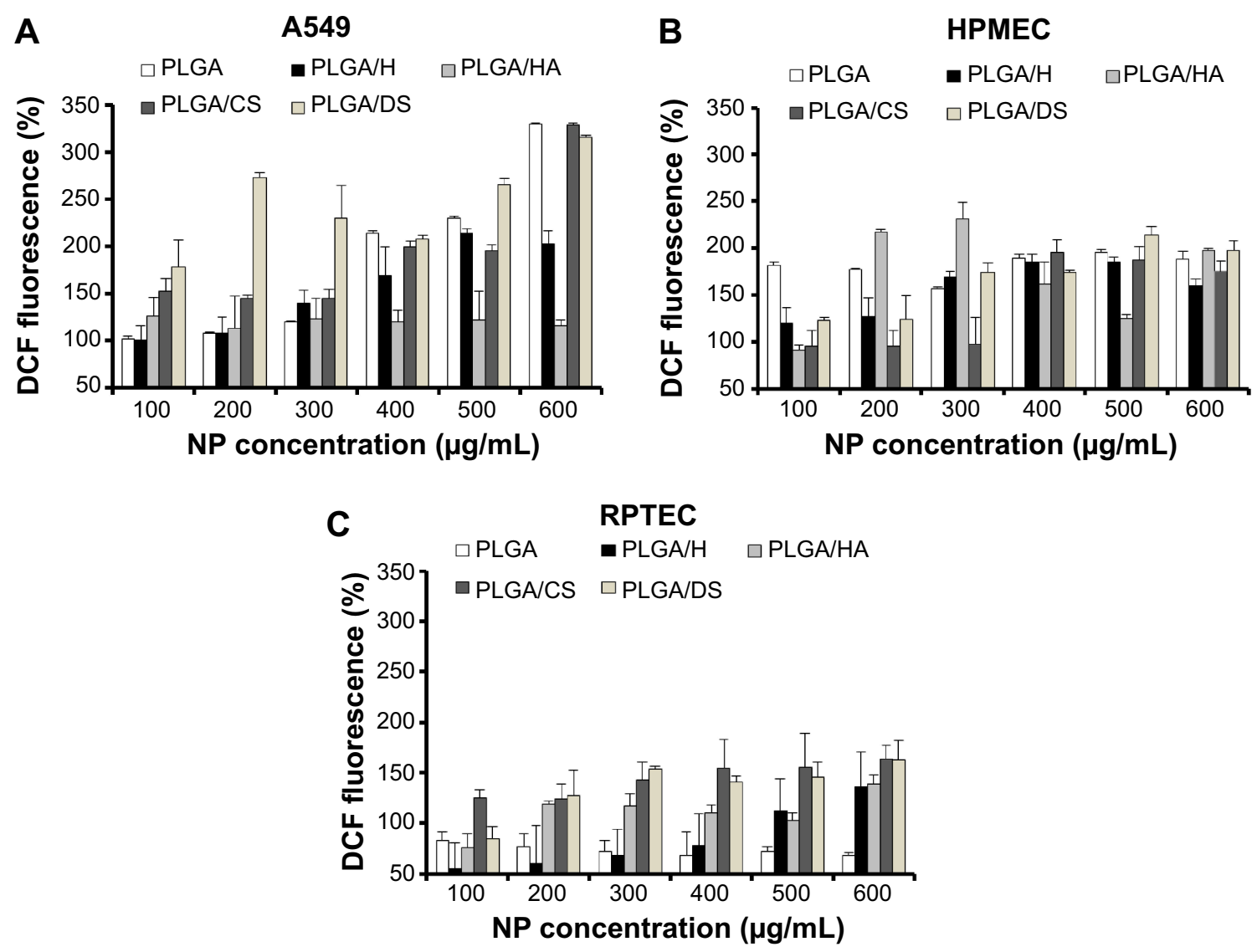

Figure 6 Reactive oxygen species induction in (A) lung epithelial adenocarcinoma (A549) cells, (B) human pulmonary microvascular endothelial cells (HPMEC), and (C) renal proximal tubular epithelial cells (RPTEC), following incubation with glycosaminoglycan-functionalized poly-lactide-co-glycolide (PLGA) nanoparticles (NPs) for 24 hours $(n=3)$.

Abbreviations: DCF, dichlorofluorescein; PLGA/CS, poly-lactide-co-glycolide functionalized with chondroitin sulfate; PLGA/DS, poly-lactide-co-glycolide functionalized with dermatan sulfate; PLGA/H, poly-lactide-co-glycolide functionalized with heparin; PLGA/HA, poly-lactide-co-glycolide functionalized with hyaluronic acid.

A

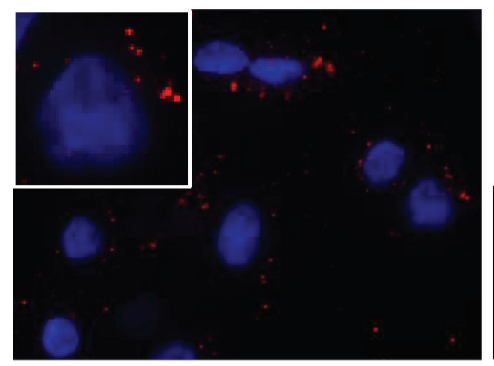

PLGA/CS

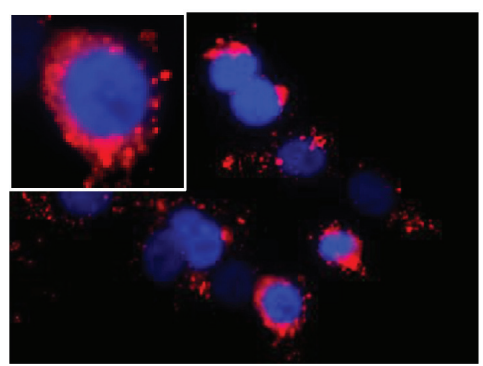

PLGA/H

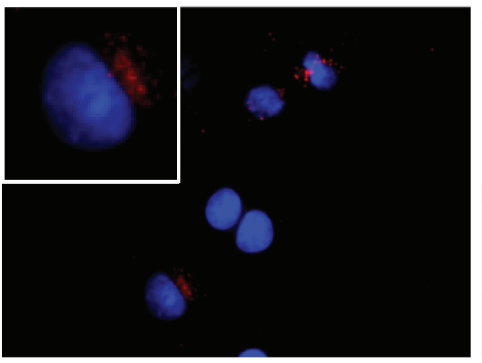

PLGA/DS

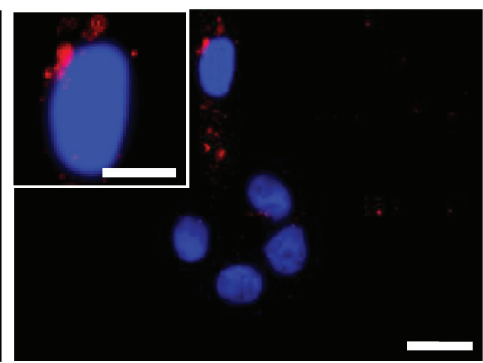


B

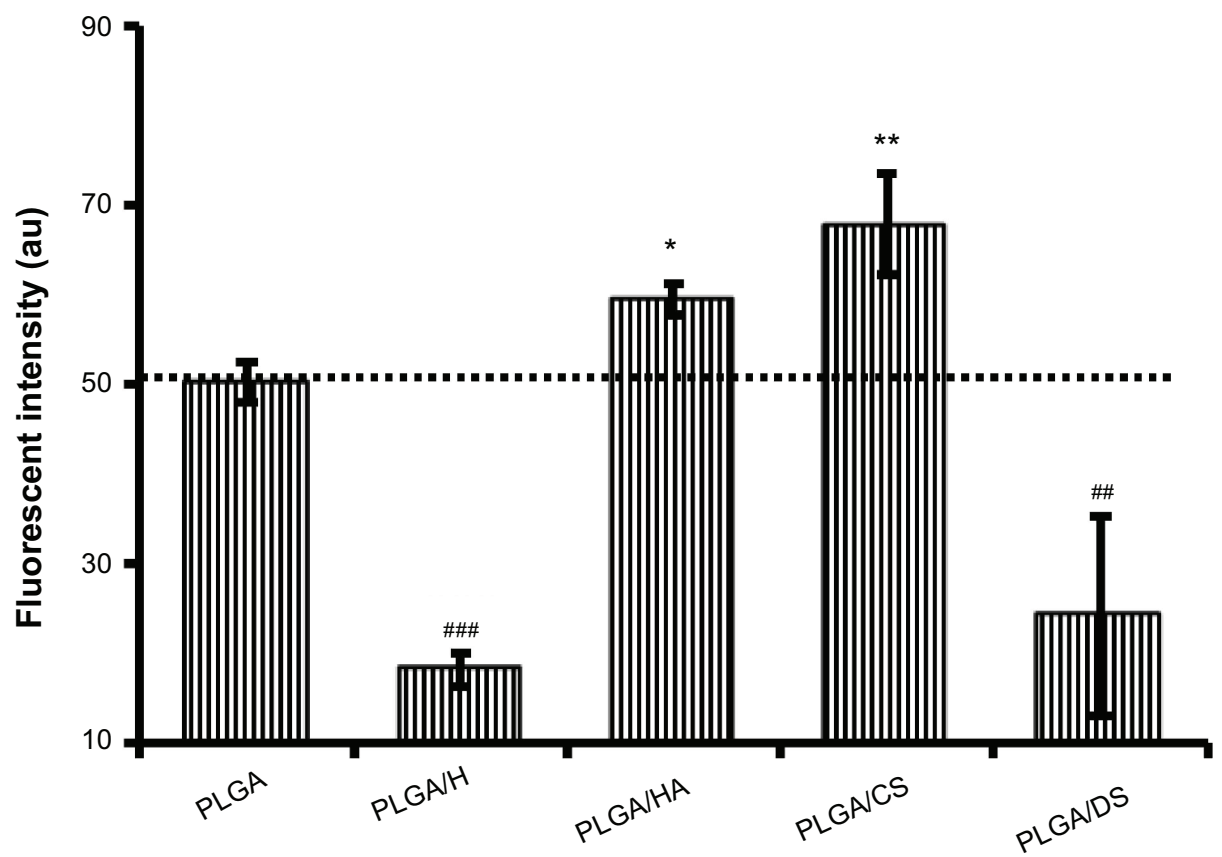

Figure 7 Fluorescent micrographs (A) and fluorescence-activated cell-sorting histogram (B) demonstrating uptake of glycosaminoglycan-functionalized poly-lactide-coglycolide (PLGA) nanoparticles by lung epithelial adenocarcinoma (A549) cells after 4 hours of incubation $(n=3)$.

Notes: $* P<0.05$ between PLGA and PLGA/HA. $* * P<0.005$ between PLGA and PLGA/CS. $P<0.005$ between PLGA and PLGA/DS. $P<0.0005$ between PLGA and PLGA/H. Scale bar: $20 \mu \mathrm{m}$ for low magnification and $10 \mu \mathrm{m}$ for high magnification (inset).

Abbreviations: DCF, dichlorofluorescein; PLGA/CS, poly-lactide-co-glycolide functionalized with chondroitin sulfate; PLGA/DS, poly-lactide-co-glycolide functionalized with dermatan sulfate; PLGA/H, poly-lactide-co-glycolide functionalized with heparin; PLGA/HA, poly-lactide-co-glycolide functionalized with hyaluronic acid.

A

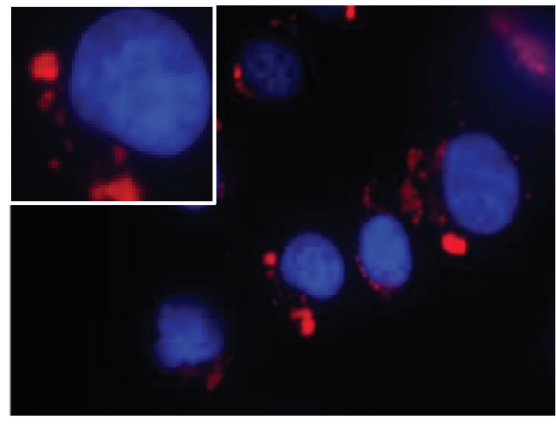

PLGA/H

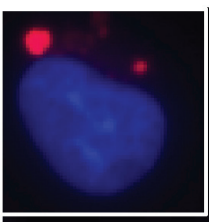

PLGA/CS

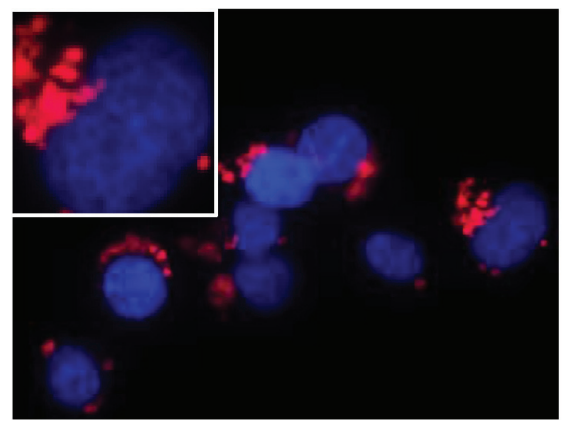

PLGA/DS

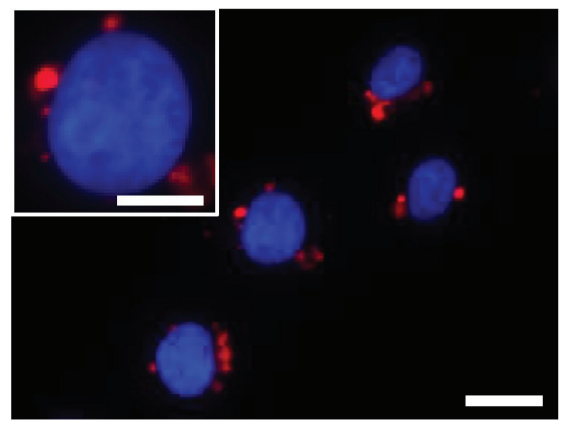

PLGA/HA

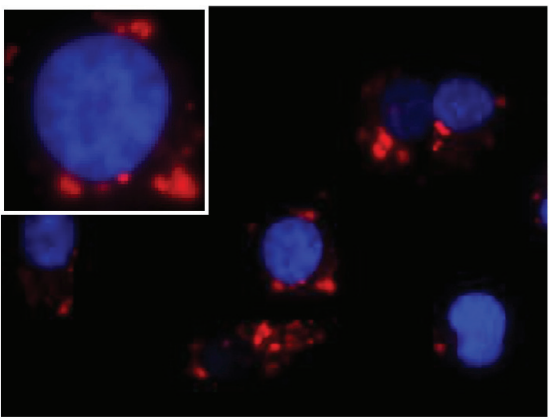

Figure 8 (Continued) 
B

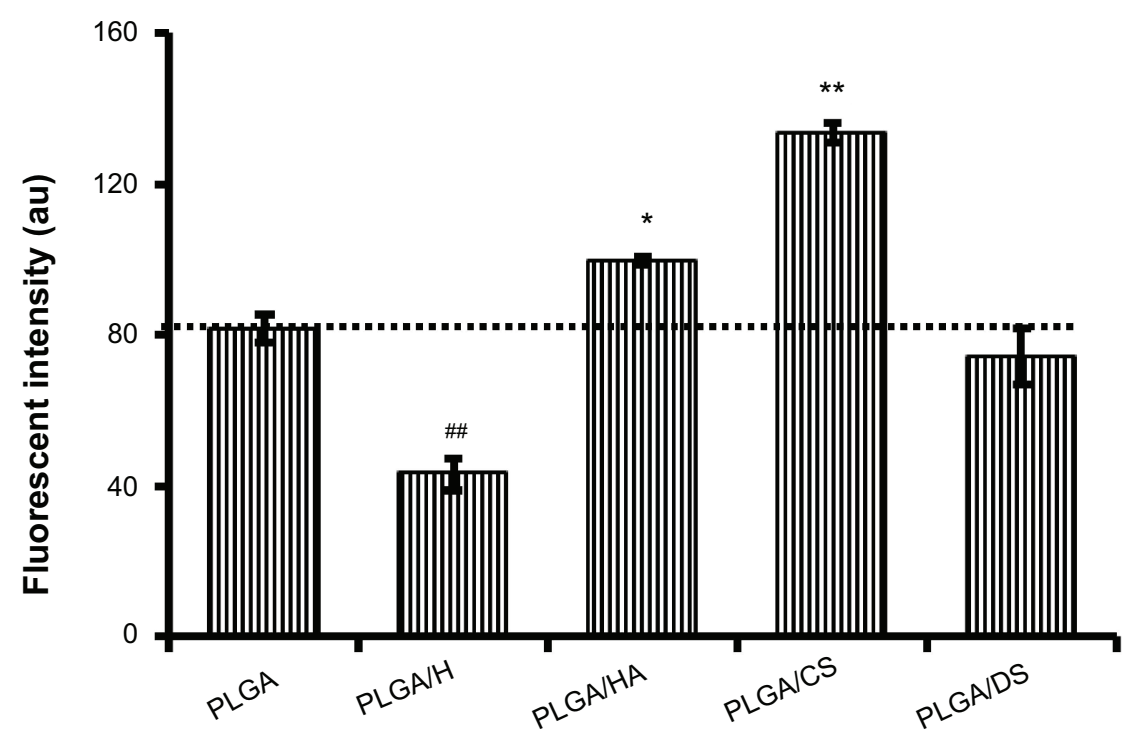

Figure 8 Fluorescent micrographs (A) and fluorescence-activated cell-sorting histogram (B) demonstrating uptake of glycosaminoglycan-functionalized poly-lactide-coglycolide (PLGA) nanoparticles (NPs) by human pulmonary microvascular endothelial cells (HPMEC) after 4 hours of incubation ( $n=3$ ).

Notes: $* P<0.05$ between PLGA and PLGA/HA. $* * P<0.005$ between PLGA and PLGA/CS. $P<0.005$ between PLGA and PLGA/H. Scale bar: $20 \mu m$ for low magnification and $10 \mu \mathrm{m}$ for high magnification (inset).

Abbreviations: PLGA/CS, poly-lactide-co-glycolide functionalized with chondroitin sulfate; PLGA/DS, poly-lactide-co-glycolide functionalized with dermatan sulfate; $\mathrm{PLGA} / \mathrm{H}$, poly-lactide-co-glycolide functionalized with heparin; PLGA/HA, poly-lactide-co-glycolide functionalized with hyaluronic acid.

case of HPMEC, the enhanced uptake observed with HA NPs may also be due to specific receptors for HA - that is, the HA receptor for endocytosis (HARE), also referred to as "Stabilin-2" (encoded by the gene STAB2) and "FEEL-2" 32 that are expressed by endothelial cells.

In analyzing these findings, it is important to note that the surface concentration of the GAGs can play an important role in altering cellular interactions and uptake. Further studies need to be carried out to develop semi-empirical relationships between GAG functionalization and cellular uptake. Nevertheless, these results taken in sum suggest that GAGs can play a role in controlling NP uptake, and provide confirmation for the hypothesis of this study. While the exact pathway of uptake needs to be further elucidated using several cell systems, the fact that the NPs were mostly concentrated in the perinuclear region is indicative of a predominantly endocytic uptake pathway. ${ }^{13,33,34}$ The findings of this study therefore indicate that GAG-functionalized PLGA NPs have potential as nanocarriers for the delivery of cancer therapeutics.

\section{Conclusion}

PLGA NPs functionalized with H, HA, CS, and DS GAGs were synthesized using a single-step modified nanoprecipitation process. The presence of GAG on the NP surface was verified by $\mathrm{p} I_{\mathrm{e}}$ measurements of the NP surface and XPS analysis. The cytocompatibility of GAG-modified PLGA NPs was assessed in three cell systems: A549 cells, HPMEC, and RPTEC, using MTT assay, LDH release, and induction of ROS. Overall, GAG-functionalized NPs showed very good cytocompatibility and did not invoke any adverse response in any of the cell systems investigated. Moreover, the particles underwent cellular internalization to differing extents in A549 cells and HPMEC and offer a potential platform for introducing a bias in the cellular uptake of PLGA NPs.

Based on these findings, GAG-functionalized PLGA NPs are promising delivery vehicles to the tumor microenvironment. However, to fully exploit the targeting potential of these NPs, their uptake mechanism in various cell systems needs to be fully understood and their biodistribution in the absence and presence of a tumor burden needs to be evaluated.

\section{Acknowledgments}

The authors would like to thank Dr Ralf Thomann, University of Freiburg, for TEM. This work was funded by the INTERREG IV Upper Rhine program (A21 NANO@MATRIX), the Excellence Initiative of the German Federal and State Governments Grant EXC 294, and the Helmholtz Virtual Institute on Multifunctional Biomaterials for Medicine.

\section{Disclosure}

The authors declare no conflicts of interest in this work. 


\section{References}

1. Siegel R, Naishadham D, Jemal A. Cancer statistics, 2013. CA Cancer J Clin. 2013;63(1):11-30.

2. Torchilin VP, Lukyanov AN. Peptide and protein drug delivery to and into tumors: challenges and solutions. Drug Discov Today. 2003;8(6):259-266.

3. Liu Y, Miyoshi H, Nakamura M. Nanomedicine for drug delivery and imaging: a promising avenue for cancer therapy and diagnosis using targeted functional nanoparticles. Int $J$ Cancer. 2007;120(12):2527-2537.

4. Torchilin V. Tumor delivery of macromolecular drugs based on the EPR effect. Adv Drug Delivery Rev. 2011;63(3):131-135.

5. Hans ML, Lowman AM. Biodegradable nanoparticles for drug delivery and targeting. Curr Opin Solid State Mater Sci. 2002;6(4):319-327.

6. Davis ME, Chen ZG, Shin DM. Nanoparticle therapeutics: an emerging treatment modality for cancer. Nat Rev Drug Discov. 2008;7(9):771-782.

7. Huwyler J, Drewe J, Krähenbühl S. Tumor targeting using liposomal antineoplastic drugs. Int J Nanomed. 2008;3(1):21-29.

8. van Vlerken LE, Vyas TK, Amiji MM. Poly(ethylene glycol)-modified nanocarriers for tumor-targeted and intracellular delivery. Pharm Res. 2007;24(8):1405-1414.

9. Sussman EM, Clarke MB Jr, Shastri VP. Single-step process to produce surface-functionalized polymeric nanoparticles. Langmuir. 2007;23(24):12275-12279.

10. Oswald S, Baunack S. Application of XPS and factor analysis for nonconducting materials. Surf Interface Anal. 1997;25(12):942-947.

11. Sasisekharan R, Shriver Z, Venkataraman G, Narayanasami U. Roles of heparan-sulphate glycosaminoglycans in cancer. Nat Rev Cancer. 2002;2(7):521-528.

12. Suh KY, Yang JM, Khademhosseini A, et al. Characterization of chemisorbed hyaluronic acid directly immobilized on solid substrates. J Biomed Mater Res Part B Appl Biomater. 2005;72(2):292-298.

13. Schipper ML, Iyer G, Koh AL, et al. Particle size, surface coating, and PEGylation influence the biodistribution of quantum dots in living mice. Small. 2009;5(1):126-134.

14. Kulkarni S, Feng SS. Effects of particle size and surface modification on cellular uptake and biodistribution of polymeric nanoparticles for drug delivery. Pharm Res. 2013;30(10):2512-2522.

15. Brigham KL. Oxidant stress and adult respiratory distress syndrome. Eur Respir J. 1990;11:482s-484s.

16. Kelly J, Moore T, Babal P, Diwan A, Stevens T, Thompson W. Pulmonary microvascular and macrovascular endothelial cells: differential regulation of $\mathrm{Ca}^{+}$and permeability. Am J Physiol. 1998;274(5):L810-L819.

17. Moore TM, Chetham PM, Kelly JJ, Stevens T. Signal transduction and regulation of lung endothelial cell permeability. Interaction between calcium and cAMP. Am J Physiol. 1998;275(2):L203-L222.

18. van Kooten C, van der Linde X, Woltman AM, van Es LA, Daha MR. Synergistic effect of interleukin-1 and CD40L on the activation of human renal tubular epithelial cells. Kidney Int. 1999;56(1):41-51.
19. Grabowski N, Hillaireau H, Vergnaud J, et al. Toxicity of surfacemodified PLGA nanoparticles toward lung alveolar epithelial cells. Int J Pharm. 2013;454(2):686-694.

20. Chung YI, Kim JC, Kim YH, et al. The effect of surface functionalization of PLGA nanoparticles by heparin- or chitosan-conjugated Pluronic on tumor targeting. J Control Release. 2010;143(3):374-382.

21. Arya N, Arora A, Vasu K, Sood AK, Katti DS. Combination of single walled carbon nanotubes/graphene oxide with paclitaxel: a reactive oxygen species mediated synergism for treatment of lung cancer. Nanoscale. 2013;5(7):2818-2829.

22. Lewinski N, Colvin V, Drezek R. Cytotoxicity of nanoparticles. Small. 2008;4(1):26-49.

23. Vandebriel RJ, De Jong WH. A review of mammalian toxicity of $\mathrm{ZnO}$ nanoparticles. Nanotechnol Sci Appl. 2012;5:61-71.

24. Sosa V, Moliné T, Somoza R, Paciucci R, Kondoh H, Lleonart ME. Oxidative stress and cancer: An overview. Ageing Res Rev. 2013 12(1):376-390.

25. Benz CC, Yau C. Ageing, oxidative stress and cancer: paradigms in parallax. Nat Rev Cancer. 2008;8(11):875-879.

26. Valko M, Rhodes CJ, Moncol J, Izakovic M, Mazur M. Free radicals, metals and antioxidants in oxidative stress-induced cancer. Chem Biol Interact. 2006;160(1):1-40.

27. Apopa P, Qian Y, Shao R, et al. Iron oxide nanoparticles induce human microvascular endothelial cell permeability through reactive oxygen species production and microtubule remodeling. Part Fibre Toxicol. 2009;6:1.

28. Alexandre J, Batteux F, Nicco C, et al. Accumulation of hydrogen peroxide is an early and crucial step for paclitaxel-induced cancer cell death both in vitro and in vivo. Int J Cancer. 2006;119(1):41-48.

29. Hiscox S, Jiang W. Regulation of endothelial CD44 expression and endothelium-tumour cell interactions by hepatocyte growth factor/ scatter factor. Biochem Biophys Res Commun. 1997;233(1):1-5.

30. Griffioen AW, Coenen MJ, Damen CA, et al. CD44 is involved in tumor angiogenesis; an activation antigen on human endothelial cells. Blood. 1997;90(3):1150-1159.

31. Penno MB, August JT, Baylin SB, et al. Expression of CD44 in human lung tumors. Cancer Res. 1994;54(5):1381-1387.

32. Harris EN, Kyosseva SV, Weigel JA, Weigel PH. Expression, processing, and glycosaminoglycan binding activity of the recombinant human 315-kDa hyaluronic acid receptor for endocytosis (HARE). J Biol Chem. 2007;282(5):2785-2797.

33. Kim SH, Jeong JH, Chun KW, Park TG. Target-specific cellular uptake of PLGA nanoparticles coated with poly(L-lysine)-poly(ethylene glycol)-folate conjugate. Langmuir. 2005;21(19):8852-8857.

34. Lai SK, Hida K, Man ST, et al. Privileged delivery of polymer nanoparticles to the perinuclear region of live cells via a non-clathrin, nondegradative pathway. Biomaterials. 2007;28(18):2876-2884. 


\section{Supplementary materials}

Table SI Size distribution of glycosaminoglycan (GAG)-functionalized poly-lactide-co-glycolide (PLGA) nanoparticles (NPs) $(0.45 \mathrm{mg} / \mathrm{mL}$ of GAG) as determined by dynamic light scattering

\begin{tabular}{lll}
\hline NP group & NP size, $\mathbf{n m}$ & PDI \\
\hline PLGA & $140 \pm 9.76$ & $0.13 \pm 0.01$ \\
PLGA/H & $125.00 \pm 10.94$ & $0.12 \pm 0.02$ \\
PLGA/HA & $132.73 \pm 10.39$ & $0.17 \pm 0.07$ \\
PLGA/CS & $128.18 \pm 7.11$ & $0.15 \pm 0.02$ \\
PLGA/DS & $134.33 \pm 9.07$ & $0.12 \pm 0.01$ \\
\hline
\end{tabular}

Abbreviations: PDI, polydispersity index; PLGA/CS, poly-lactide-co-glycolide functionalized with chondroitin sulfate; PLGA/DS, poly-lactide-co-glycolide functionalized with dermatan sulfate; PLGA/H, poly-lactide-co-glycolide functionalized with heparin; PLGA/HA, poly-lactide-co-glycolide functionalized with hyaluronic acid.
Table S2 Size distribution of glycosaminoglycan (GAG)-functionalized poly-lactide-co-glycolide (PLGA) nanoparticles (NPs) $(0.45 \mathrm{mg} / \mathrm{mL}$ of $\mathrm{GAG})$ as determined by transmission electron microscopy

\begin{tabular}{ll}
\hline NP group & NP size, $\mathbf{n m}$ \\
\hline PLGA & $86.08 \pm 26.39$ \\
PLGA/H & $100.00 \pm 24.46$ \\
PLGA/HA & $101.73 \pm 17.78$ \\
PLGA/CS & $101.18 \pm 26.33$ \\
PLGA/DS & $97.33 \pm 23.87$ \\
\hline
\end{tabular}

Abbreviations: PLGA/CS, poly-lactide-co-glycolide functionalized with chondroitin sulfate; PLGA/DS, poly-lactide-co-glycolide functionalized with dermatan sulfate; PLGA/H, poly-lactide-co-glycolide functionalized with heparin; PLGA/HA, polylactide-co-glycolide functionalized with hyaluronic acid.

Table S3 Binding energy of functional groups of glycosaminoglycan-functionalized poly-lactide-co-glycolide (PLGA) nanoparticles as determined by X-ray photoelectron spectroscopy

\begin{tabular}{lllllll}
\hline Bond type & PLGA & PLGA/H & PLGA/HA & PLGA/DS & PLGA/CS & Reference (eV) \\
\hline C-H, C-C $(\mathrm{eV})$ & 285.1 & 284.7 & 284.6 & 284.8 & 284.7 & $284.6-285.0$ \\
C-N/C-S $(\mathrm{eV})$ & - & 285.5 & 285.3 & 285.5 & 285.4 & $284.5-286.1(\mathrm{C}-\mathrm{N})$ \\
& & & & & & and $284-284.3(\mathrm{C}-\mathrm{S})$ \\
$\mathrm{C}-\mathrm{O}-\mathrm{C}(\mathrm{eV})$ & 287.0 & 286.9 & 287.0 & 287.0 & 286.9 & 287.0 \\
O-C=O (eV) & 289.1 & 289.1 & 289.2 & 289.1 & 289.1 & 289.0 \\
\hline
\end{tabular}

Abbreviations: PLGA/CS, poly-lactide-co-glycolide functionalized with chondroitin sulfate; PLGA/DS, poly-lactide-co-glycolide functionalized with dermatan sulfate; PLGA/H, poly-lactide-co-glycolide functionalized with heparin; PLGA/HA, poly-lactide-co-glycolide functionalized with hyaluronic acid.

PLGA

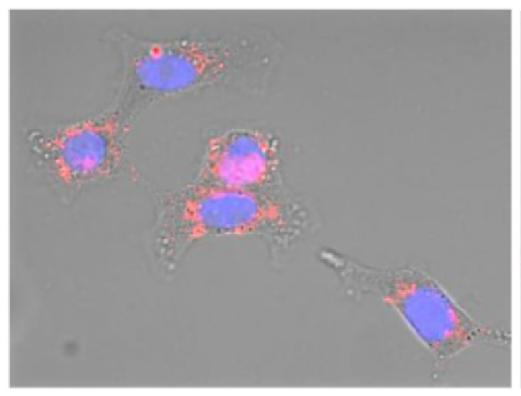

PLGA/CS

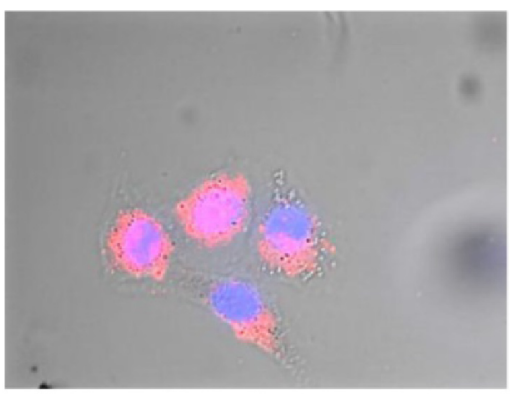

PLGA/H

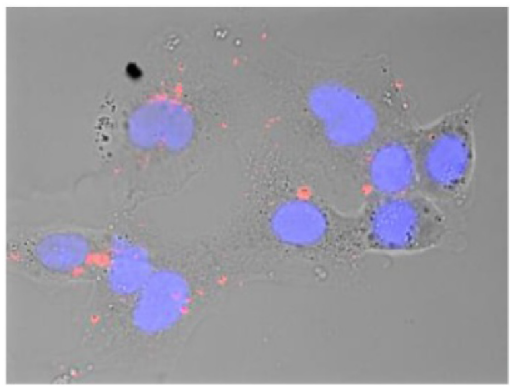

PLGA/HA

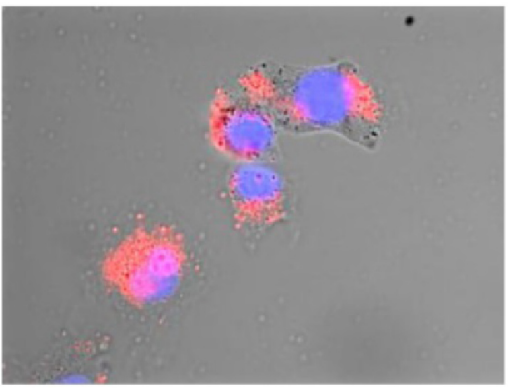

PLGA/DS

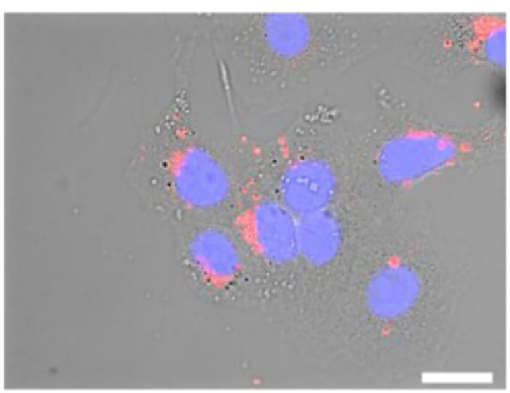

Figure SI Uptake of glycosaminoglycan-functionalized poly-lactide-co-glycolide (PLGA) nanoparticles by lung epithelial adenocarcinoma (A549) cells after 4 hours of incubation as depicted by overlay of differential interference contrast and fluorescence images.

Note: Scale bar: $20 \mu \mathrm{m}$.

Abbreviations: PLGA/CS, poly-lactide-co-glycolide functionalized with chondroitin sulfate; PLGA/DS, poly-lactide-co-glycolide functionalized with dermatan sulfate; PLGA/H, poly-lactide-co-glycolide functionalized with heparin; PLGA/HA, poly-lactide-co-glycolide functionalized with hyaluronic acid. 
PLGA

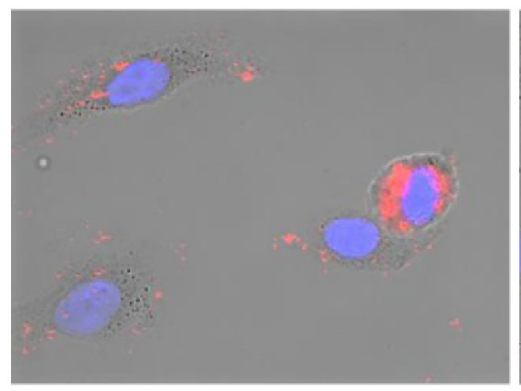

PLGA/H

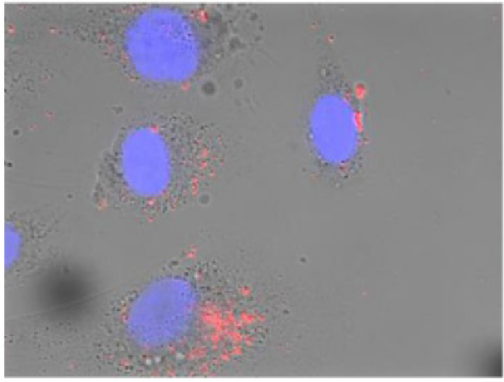

PLGA/HA

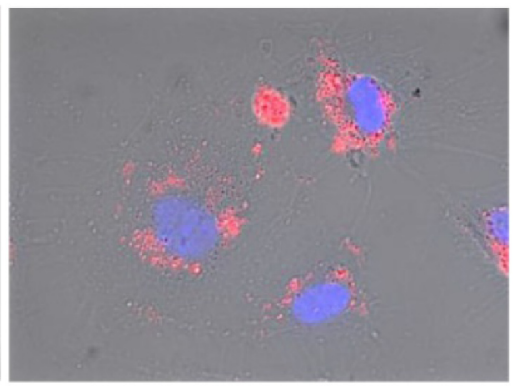

PLGA/CS

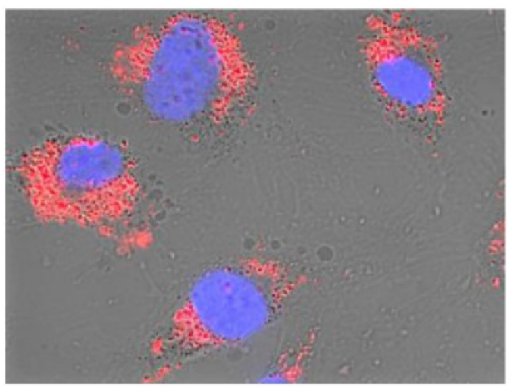

PLGA/DS

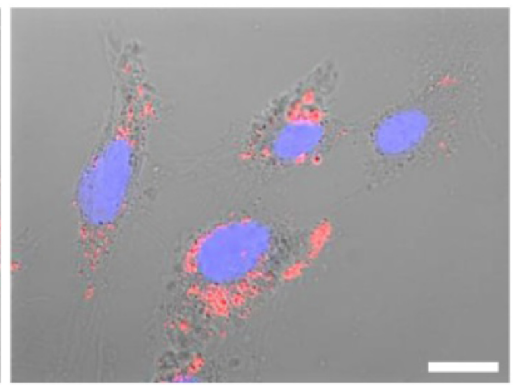

Figure S2 Uptake of glycosaminoglycan-functionalized poly-lactide-co-glycolide (PLGA) nanoparticles by human pulmonary microvascular endothelial cells after 4 hours of incubation as depicted by overlay of differential interference contrast and fluorescence images.

Note: Scale bar: $20 \mu \mathrm{m}$.

Abbreviations: PLGA/CS, poly-lactide-co-glycolide functionalized with chondroitin sulfate; PLGA/DS, poly-lactide-co-glycolide functionalized with dermatan sulfate; $\mathrm{PLGA} / \mathrm{H}$, poly-lactide-co-glycolide functionalized with heparin; PLGA/HA, poly-lactide-co-glycolide functionalized with hyaluronic acid.

\section{Publish your work in this journal}

The International Journal of Nanomedicine is an international, peerreviewed journal focusing on the application of nanotechnology in diagnostics, therapeutics, and drug delivery systems throughou the biomedical field. This journal is indexed on PubMed Central,

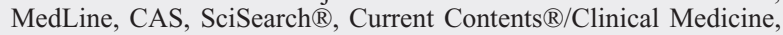

Journal Citation Reports/Science Edition, EMBase, Scopus and the Elsevier Bibliographic databases. The manuscript management system is completely online and includes a very quick and fair peer-review system, which is all easy to use. Visit http://www.dovepress.com/ testimonials.php to read real quotes from published authors.

\footnotetext{
Submit your manuscript here: http://www.dovepress.com/international-journal-of-nanomedicine-journal
} 